\title{
Análisis de propuestas curriculares dominicanas para lectoescritura inicial de la población estudiantil con necesidades específicas de apoyo educativo
}

Analysis of Dominican curricular approaches for early grade literacy of student population with specific needs of learning support

\section{Volumen 22, Número 1 \\ Enero - Abril \\ pp. $1-26$}

Irene Altagracia Pérez-García

Winny Plasencia-Peralta

\section{Citar este documento según modelo APA}

Pérez-García, Irene Altagracia., y Plasencia-Peralta, Winny. (2022). Análisis de propuestas curriculares dominicanas para lectoescritura inicial de la población estudiantil con necesidades específicas de apoyo eductivo. Revista Actualidades Investigativas en Educación, 22(1), 1-26. Doi. https://doi.org/10.15517/aie.v22i1.49072 


\title{
Análisis de propuestas curriculares dominicanas para lectoescritura inicial de la población estudiantil con necesidades específicas de apoyo educativo
}

Analysis of Dominican curricular approaches for early grade literacy of student population with specific needs of learning support

\author{
Irene Altagracia Pérez-García ${ }^{1}$ \\ Winny Plasencia-Peralta²
}

\begin{abstract}
Resumen: Esta investigación se enmarca en el paradigma de la educación inclusiva. Para cumplir con este derecho, el Ministerio de Educación (MINERD) propone una serie de ajustes curriculares individualizados (ACl) con la intención de favorecer la inclusión de la población estudiantil con necesidades específicas de apoyo educativo (NEAE) que asiste a escuelas regulares para adquirir las competencias necesarias en su desarrollo integral. Se examinó cuán alineados están el Diseño Curricular dominicano y las guías de $\mathrm{ACl}$ con respecto a la evidencia recopilada acerca de la lectoescritura inicial (LEI) en la población estudiantil con NEAE. Para la realización de este estudio de tipo cualitativo de corte transversal y de carácter descriptivo, se realizó una revisión bibliográfica, luego se determinaron los documentos que se analizaron mediante un árbol de categorías. Con la finalidad de complementar este proceso, se realizaron 13 entrevistas semiestructuradas a las personas encargadas de la creación y difusión de las guías en cuestión, los datos fueron procesados en MAXQDA 2020, por último, se trianguló la información. El levantamiento de datos ocurrió durante el primer cuatrimestre del año 2021. No en todos los documentos, objeto del estudio, están presentes las categorías de análisis, y las guías de ajustes curriculares no abordan la adquisición de la LEI en la población con NEAE. El estudio concluye que los documentos se corresponden parcialmente con lo planteado por la evidencia y que se requiere una propuesta de ajustes curriculares que responda a las necesidades específicas de la población infantil.
\end{abstract}

Palabras clave: guía de estudios, alfabetización, educación básica, necesidades educacionales.

\begin{abstract}
This research focuses on the inclusive education paradigm. To comply with the right of inclusive education, the Dominican Ministry of Education proposes a series of individualized curricular adjustments to support that student population with specific needs of learning support attending regular schools learn the necessary skills for their social development and are treated with equity. This study seeks to determine how aligned are the Dominican Curriculum Design and the individualized curricular adjustment guides with the evidence related to early grade literacy in student population with temporary educational needs. The design of this research The study is qualitative, cross-sectional, and descriptive. A literature review was carried out and selected the documents to be analyzed through a category tree. To complement the analysis of the texts, semi-structured interviews were conducted with people responsible for the creation and dissemination of the guides that were studied; data was processed with MAXQDA 2020, and then the information triangulated, the data collection occurred during the first four months of the year 2021. Results showed that many categories analyzed were absent in the documents and that the curricular adjustment guides do not address the acquisition of literacy in the specific needs of learning support population. The study concludes that the documents partially correspond to the evidence and that a proposal for curricular adjustments that responds to the specific needs of the child population is required.
\end{abstract}

Keywords: curriculum guides; literacy; basic education; educational needs.

1 Técnica distrital del Ministerio de Educación de la República Dominicana, Santiago, República Dominicana. Especialista en Lectura y Escritura de la Pontificia Universidad Católica Madre y Maestra. Dirección electrónica: irene.perezg05@gmail.com ORCID: https://orcid.org/0000-00027973-6672

2 Docente del Ministerio de Educación de la República Dominicana, Santiago, República Dominicana. Colaboradora de la Academia Dominicana de la Lengua. Licenciada en Educación mención Filosofía y Letras en Universidad Autónoma de Santo Domingo. Dirección electrónica: winnypp0606@gmail.com ORCID: https://orcid.org/0000-0001-8223-4617

Artículo recibido: 02 de setiembre, 2021

Enviado a corrección: 13 de octubre, 2021

Aprobado: 22 de noviembre, 2021

Los contenidos de este artículo están bajo una licencia Creative Commons 


\section{Introducción}

Las necesidades específicas de apoyo educativo (NEAE) se evidencian cuando las barreras dificultan que una persona acceda los aprendizajes y por consecuencia, se requieran medios o recursos distintos y atención especial (Dabdub-Moreira y Pineda-Cordero, 2015; López y Valenzuela, 2015; Luque, 2009; UNESCO, 2017). López y Valenzuela (2015) indican que las NEAE pueden manifestarse en un momento y extenderse, sin permanecer toda la vida. Dentro de esta subclasificación se encuentran las necesidades asociadas a la adquisición de la Lectoescritura Inicial (LEI). En la mayor parte de los casos, estas no dependen de un diagnóstico clínico, su detección se media desde el aula (Ocampo y Calero, 2018); esta es una de las razones por la que este estudio se enfoca en ellas. Un hito en la historia de las NEAE es que a esta población se le reconociera el derecho de asistir a centros de enseñanza regular (Warnock, 1978).

En las últimas décadas se ha producido un debate entre quienes atribuyen un enfoque clínico a la terminología necesidades educativas especiales (NEE) y aquellos que la asumen como inclusiva; pese a esto, en múltiples países NEE y NEAE aún se emplean indistintamente (Robson, 2005). Las investigaciones más recientes señalan que NEAE tiene un enfoque en las barreras y su eliminación, más que en las dificultades de la población estudiantil para aprender; asimismo, las NEAE parten de evaluaciones psicoeducativas que evitan diagnósticos y segregación, por medio de la valoración de diferencias individuales (Luque y Luque, 2015; Ruiz, 2020). Por razones históricas y funcionales, las fuentes consultadas en este artículo responden tanto a NEE como NEAE, sin embargo, debido al paradigma inclusivo y su concepción de la población estudiantil se asumen las NEAE como filosofía. En el caso particular de República Dominicana, después de publicarse la Ordenanza 01-2018, se acuñó la expresión NEAE en todos los documentos posteriores al año 2018.

Existen paradigmas que, con frecuencia, rigen el quehacer en las escuelas, estos son exclusión, segregación, integración e inclusión. Warnock (1978) y la UNESCO (2019) plantean que el principio de educar junta a la población estudiantil con y sin necesidades específicas de apoyo educativo sustenta el derecho a la autorrealización y evita la exclusión de las aulas, lo cual constituye un derecho fundamental. Para lograrlo se requiere hablar de inclusión, que implica ajustes a los postulados del currículo, con el fin de conseguir el máximo desarrollo de las capacidades de LEI de la población estudiantil mediante el respeto de las diferencias individuales que presentan. Estos ajustes incluyen, por ejemplo, el desarrollo de guías de ajustes curriculares que promuevan la inclusión a través de la implementación de las 
modificaciones avaladas al documento curricular base que promueve la homogeneización del perfil de egreso (Guirado y Guerra, 2013).

En República Dominicana, la propuesta nacional recomienda un ambiente inclusivo con atención a la diversidad, que favorezca el acceso universal a educación de calidad y sin discriminación (Ministerio de Educación de la República Dominicana, 2016a; Stainback y Stainback, 1999). En ese sentido, el Ministerio de Educación de la República Dominicana (MINERD) (2018) ha elaborado propuestas complementarias al currículo dominicano con la intención de desarrollar las competencias comunicativas de la población estudiantil con NEAE del sistema educativo. Estas propuestas se encuentran en el marco de la Ley No. 5-13 sobre discapacidad en la República Dominicana, la cual vela por la igualdad de oportunidades de la población. Asimismo, a través de la Ordenanza 04-2018, se norman los servicios y estrategias para el estudiantado con NEAE acordes al currículo establecido. También la Ordenanza 022016 que establece el sistema de evaluación de los aprendizajes en la educación inicial y primaria en correspondencia con el currículo revisado y actualizado. Además, el MINERD (2018) propone tres documentos para la enseñanza de la LEI de la población estudiantil con NEAE: la guía de ajustes curriculares titulada Orientaciones Generales para la Atención a la Diversidad, Guía para la Realización de Ajustes Curriculares Individualizados (ACl). También las Bases de la Revisión y Actualización Curricular (MINERD, 2016b) y el Diseño Curricular Dominicano, Nivel Primario, Primer Ciclo (MINERD, 2016a).

A pesar del planteamiento en leyes, con frecuencia, no se alcanzan los resultados esperados, especialmente en la adquisición de la lengua escrita y los procesos de comprensión y producción a lo largo de la formación académica (Castillo y Mola, 2016; León, 2018). En reconocimiento de la importancia de la inclusión de la población con NEAE, se propone el presente estudio que busca responder ¿Cuán alineados se encuentran el currículo y sus guías de ajuste curricular con lo que indica la evidencia respecto a la LEI de la población estudiantil con NEAE? Para lograrlo se ha analizado la coherencia de las guías de ajustes curriculares y el currículo en la LEI de la población estudiantil con NEAE que cursa los tres primeros grados de la educación primaria frente a la evidencia científica. Esta investigación contribuye a la discusión sobre las áreas de mejora de las propuestas curriculares y el análisis de las modificaciones que son necesarias para responder a las necesidades educativas de toda la población estudiantil dominicana. 


\section{Referente teórico}

En esta sección se presenta la delimitación teórica del trabajo. Las NEAE que ocupan esta investigación están relacionadas con las dificultades en la adquisición de la LEI que pueden manifestarse tanto en el plano oral (hablar y escuchar) como en el escrito (leer y escribir) evidenciados en un desempeño bajo en las funciones del lenguaje sin vinculación a una discapacidad intelectual (López y Valenzuela, 2015). A lo largo de los siguientes apartados se desarrollarán los paradigmas presentes de las propuestas curriculares para la población estudiantil con NEAE, la concepción de herramientas didácticas para la enseñanza a la población estudiantil con NEAE y el proceso de LEl de la población estudiantil con NEAE desde un enfoque funcional y comunicativo (enfoque que sustenta el currículo dominicano).

\subsection{Paradigmas presentes en las propuestas curriculares para la población estudiantil con NEAE}

El reconocimiento del derecho a la educación de la población estudiantil con NEAE permitió su participación en aulas ordinarias según lo establece el Diseño Universal del Aprendizaje (DUA) (Arnaiz, 2012; Valencia-Pérez y Hernández-Gonzalez, 2017). Esto representó un paso significativo desde la segregación hacia la integración. Eventualmente, para incluir a la población estudiantil, la escuela asumió cambios, uno de los más significativos y permanentes, a nivel nacional, hasta el momento. Es el uso de guías de ajustes curriculares.

Con el fin de lograr el cumplimiento del derecho a la educación de las personas con NEAE se ha evolucionado en paradigmas, lo que ha tenido implicaciones en las políticas educativas y en la implementación en los centros escolares. Los principales paradigmas son exclusión (no participación en la escuela), segregación (asistencia a escuelas específicas para NEAE), y los paradigmas de integración e inclusión, que surgen de la mano con el reconocimiento de derechos de la población estudiantil. La UNESCO (2008) plantea que la integración surgió como una alternativa a la segregación, con el principal propósito de que el estudiantado con NEAE acceda a escuelas comunes. Aunque la población estudiantil comparta un mismo espacio, la integración no permite las mismas oportunidades de aprendizaje cuando no existen transformaciones metodológicas significativas por parte de la escuela; esto tampoco lo facilita un currículo sin propuestas concretas para lograr una participación activa (Leiva, 2013).

La inclusión, por su parte, se ocupa de fomentar igualdad de oportunidades y acceso sin importar si se trata de una persona con NEAE o no. En este paradigma la diversidad es la 
norma y por esto se toman medidas para favorecer a todas las personas (García-Llamas, 2008; Leiva, 2013; Molina, 2015; UNESCO, 2008). Respecto a la inclusión plena, Luque (2009) afirma "de acuerdo con estos fines, los programas de integración deben seguir aplicándose, en la medida que se precisan para la compensación y distribución justa de recursos y servicios, convirtiéndose así en un mecanismo de inclusión" (p.217).

Bajo el paradigma de la inclusión, el currículo es homogéneo, flexible y debe permitir tomar medidas de atención a la diversidad y realizar ajustes para responder a cada necesidad (García-Llamas, 2008; UNESCO, 2008; Valencia-Pérez y Hernández-González, 2017; Valladares et al., 2016). Sin estas modificaciones las NEAE no pueden ser trabajadas (Brennan, 1985), pues el currículo es un referente que no puede aplicarse a toda la población estudiantil por igual (Arnaiz, 2012; Parrilla et al. 2012; Stainback y Stainback, 1999). Estas modificaciones son indispensables para la inclusión del estudiantado con NEAE y para que desde ellas se incorporen las herramientas didácticas idóneas.

\subsection{Concepción de herramientas didácticas de enseñanza para la población estudiantil con NEAE}

Se afirma que la LEI se desarrolla en los tres primeros grados de primaria, usualmente entre la edad de 6 a 8 años (Braslavsky, 2000; Chall, 1983). Esta afirmación complejiza la detección de una NEAE debido a que surge la inquietud de cómo determinarla si los expertos indican su desarrollo durante un lapso de tres años o más. Debido a que no toda la población estudiantil empieza su proceso de LEI al mismo ritmo, y la intención de la escuela es reducir esa brecha de desigualdad se hace necesario recurrir a estrategias que favorezcan el acceso de todos a esa habilidad. Frecuentemente se observa que al estudiantado con NEAE se le dificulta realizar la asociación con los signos gráficos y el léxico interior o lenguaje externo (Lores-Leyva y Calzadilla-González, 2013). La ausencia de estas habilidades, en ocasiones, se les atribuye a ambientes que no favorecen la independencia y autonomía, bajo fomento de hábitos de estudio, poco acceso a infraestructura sanitaria y de higiene, además de la falta de una alimentación adecuada para su desarrollo físico, alguna discapacidad física o dificultad de comprensión (Quiroga, 2010). En respuesta a esto, se formulan ajustes curriculares que permitan a la persona docente mediar los aprendizajes para esta población estudiantil.

Una innovación educativa que persigue sustituir los ajustes curriculares se refiere a las medidas de atención a la diversidad, que forman parte del paradigma inclusivo. Estas medidas -propuestas desde la teoría constructivista- se priorizan por encima de los ajustes debido a 
que se enfocan en las barreras externas, conciben que se debe mejorar el ambiente en lugar de visualizar a la población estudiantil desde un enfoque deficitario y clínico (Paz-Delgado y Barahona, 2020; Valencia-Pérez y Hernández-González, 2017). Además, son las herramientas que se recomiendan previo al uso de ajustes para responder al principio de que cada persona es diferente y aprende de manera diversa. Algunas de las recomendaciones para desarrollar estas medidas son: empezar desde la etapa o nivel en que se encuentre el estudiantado, construir aprendizajes significativos, promover que estos se adquieran con autonomía, fomentar que se transformen los esquemas de conocimiento y prestar mayor atención a la zona de desarrollo próximo (Quiroga, 2010). Si al desarrollar todas las medidas de atención a la diversidad, la población estudiantil con NEAE aún no evoluciona, la literatura recomienda trabajo interdisciplinario con los especialistas de la escuela seguido de referimientos externos (Corredor-Ponce, 2016; Dabdub-Moreira y Pineda-Cordero, 2015).

Específicamente en la lectoescritura se propone que la adquisición de la lengua se favorezca hacia el acceso a recursos que estimulan diversos canales -oral y escrito- y que estén adaptados, por ejemplo, tomando en cuenta tamaño y color de letras, así como el volumen en la comunicación (Espada y Moreno, 2016). Para favorecer esto, Valladares et al. (2016) afirman que "el currículum debe especificar objetivos de aprendizaje amplios, que garanticen las competencias básicas, más que contenidos detallados" (p. 38).

Otro elemento didáctico de importancia es la evaluación de los aprendizajes. GarcíaLlamas (2008) plantea que debe ser un proceso cooperativo y cíclico, que promueva la participación activa del estudiantado, además de estar alineado a los objetivos y contenidos de aprendizaje. Asimismo, es indispensable que los instrumentos de evaluación sean altamente flexibles. Al respecto, Gómez (2018) afirma que "las pruebas estandarizadas son una forma sutil de obligar al desarrollo de un currículo homogéneo para todo el mundo" (p. 99). Otro aspecto importante en el contexto de la inclusión es la medición de los resultados basada en el rendimiento individual (Stainback y Stainback, 1999). Por su parte, Puigdellívol (1998) refiere que las funciones de la evaluación son: informativa, incentivadora y formativa; las evaluaciones priorizan el aprendizaje sobre la asociación del estudiantado a un simple valor numérico. Las herramientas didácticas previamente reseñadas (evaluación, contenidos, objetivos) representan los elementos principales a tomar en consideración al momento de realizar ajustes curriculares para favorecer la inclusión. 


\subsection{El proceso de lectoescritura inicial de la población estudiantil con NEAE}

La LEI es un proceso que permite que las personas puedan desenvolverse con autonomía en el ámbito social, para que la LEI se desarrolle satisfactoriamente se debe dar una serie de condiciones, una de las más importantes es la dimensión afectiva, que se manifiesta en la interacción estudiantado - docente (MINERD, 2016b). En el proceso educativo se precisan espacios de participación activa, para esto son indispensables las prácticas de LEI contextualizadas. Otra condición de alta importancia es la relación entre pares, proceso que fomenta un mayor aprendizaje (Montealegre y Forero, 2006).

Las premisas teóricas postulan que la LEI requiere de la construcción de significados más allá de una habilidad de codificar y decodificar (Báez y D’Ottavio, 2020; Ferreiro, 2019; Quiroga, 2010; Teberosky, 2003). Una de las múltiples razones para que la población estudiantil asista a escuelas regulares es que "el conocimiento sobre la lengua escrita se construye mediante la participación de la persona en el contexto natural y cotidiano al utilizarlo de manera funcional con sentido y significados reales" (Chaves, 2001, p. 3). En lo que se refiere al currículo dominicano, MINERD (2016b) aborda la LEI como práctica social para construir significado e indica que "una persona alfabetizada es aquella que conoce un código escrito y lo descifra, y es a la vez capaz de interpretar y crear textos orales y escritos" (p. 75).

Por su parte, Jaramillo (2018) afirma que uno de los retos más comunes en la población estudiantil con NEAE está asociado a la concentración, comprensión y retención de los conceptos. En ese sentido, se hace necesario reiterar que esas son las características de las NEAE en un enfoque educativo y no clínico. Para mediar esto, Ocampo y Calero (2018) recomiendan que el estudiantado esté en contacto con materiales variados y auténticos de lectura y que cualquier docente, sin importar su área académica, se comprometa a motivar a leer y escribir a todos sus estudiantes. Otro principio que recomiendan es que todos reciban instrucciones acordes a sus ritmos y formas de aprender. Además, sugieren que las habilidades orales, de lectura y escritura se fomenten en contacto con diferentes tipos de textos. Por último, orienta que la persona docente debe ser facilitadora, mediadora y gestora de prácticas comunicativas.

En consonancia con el párrafo anterior, es preciso reiterar que el aprendizaje de la lengua se desarrolla mediante competencias específicas que, en actividades cotidianas, se traducen en hablar, escuchar, leer y escribir mediante la interacción con textos. Uno de los principios esenciales para la adquisición de la LEI es la capacidad de realizar una relación sonoro-gráfica, para esto se toma la oralidad como punto de partida, pues es la habilidad que 
la mayoría del estudiantado adquiere de manera natural. En esta transición la conciencia fonológica juega un papel preponderante, pues se concibe un cimiento oral para acceder a la escritura y el avance de las hipótesis (Quiroga, 2010). La conciencia fonológica consiste en que la población estudiantil pueda dominar los fonemas de la lengua y posteriormente asociarlos a su representación gráfica. La hipótesis silábica es el inicio de la fonetización, las demás hipótesis de escritura son: presilábica, silábico alfabética y alfabética. Un ámbito de alta importancia es concebir a la población estudiantil como sujetos activos del aprendizaje, esto favorece un reconocimiento más específico sobre lo que saben y lo que necesitan saber (Caballeros et al., 2014; Ferreiro, 2019). Los postulados expuestos se refieren a las competencias necesarias para la población estudiantil con NEAE y su aprendizaje de LEI, los autores previamente citados afirman que para lograr inclusión plena es indispensable que la población estudiantil con NEAE desarrolle su proceso de LEI en un ambiente en que predominen las medidas de atención a la diversidad, ya que solo estas aportarán una respuesta específica a sus necesidades.

\section{Metodología}

\subsection{Enfoque}

La investigación realizada es de tipo cualitativo de corte transversal y de carácter descriptivo. Se realizó con el objetivo de analizar la alineación del currículo dominicano y las guías de ajustes curriculares con respecto a la evidencia recopilada acerca de la LEI en la población estudiantil con NEAE. Para esto, se partió de una revisión bibliográfica de las principales bases de datos de Centroamérica y el Caribe, entre ellas, la biblioteca virtual de la RedLEI y universidades miembros que conforman la Red, los Ministerios de Educación de los países Centroamericanos, Organizaciones internacionales (UNESCO, UNICEF, entre otros), repositorios y revistas internacionales indexadas, además de libros y artículos científicos.

\subsection{Unidades de análisis}

Los documentos seleccionados como unidad de análisis representaron las fuentes primarias de la información. Se seleccionaron tomando en cuenta la pregunta de investigación, la revisión bibliográfica y el contexto de la investigación. La finalidad de la búsqueda fue localizar e identificar documentos y evidencias relacionados con "Diseños curriculares inclusivos", "Aplicación de ajustes curriculares en la lectoescritura inicial”, "Ajustes curriculares en la población estudiantil con necesidades específicas de apoyo educativo" para así evitar la 
omisión de variables y conseguir la validez interna y externa. Luego de realizar una búsqueda sistemática, se recopilaron más de 120 textos, entre ellos currículos de diferentes países, guías de ajustes curriculares de países centroamericanos, artículos sobre la aplicación de ajustes curriculares y libros sobre las necesidades educativas presentes durante la población estudiantil que está aprendiendo LEI. El levantamiento de información se realizó durante el primer cuatrimestre del año 2021.

El período de los documentos seleccionados es 2016-2018. A continuación se nombra cada uno de estos documentos:

- El Diseño Curricular del Nivel Primario: Primer Ciclo. (MINERD, 2016a).

- Bases de la Revisión y Actualización Curricular. (MINERD, 2016b).

- Las Orientaciones Generales para la Atención a la Diversidad, Guía para la Realización de Ajustes Curriculares Individualizados (ACI). (MINERD, 2018).

- La Ordenanza 04-2018. (Ordenanza 04-2018, 2018. Norma los servicios y estrategias para el cuerpo estudiantil con necesidades específicas de apoyo educativo acorde al currículo. 26 de julio del 2018).

- La Ordenanza 02-2016. (Ordenanza 02-2016, 2016. Establece el sistema de evaluación de los aprendizajes en la Educación inicial y primaria en correspondencia con el currículo revisado y actualizado. 19 de diciembre del 2016).

Además del análisis documental se realizaron entrevistas: los criterios de inclusión para la población entrevistada fueron: 1) formar parte de los Centros de Recursos para la Atención a la Diversidad (CAD) o de la Dirección General de Educación Especial -ambas son dependencias del MINERD-, y 2) haber participado en la creación, difusión y/o seguimiento de las guías de ajustes curriculares. Las personas integrantes residen y laboran en distintos puntos del territorio nacional dominicano y forman parte del MINERD desde antes de la elaboración de las guías de ajustes curriculares hasta la fecha de la investigación.

\subsection{Técnicas de recolección}

Las personas participantes de esta investigación debieron acceder a dos consentimientos informados: uno para permitir la grabación y otro para participar de la investigación, ambos fueron aprobados por el Comité de ética de la Universidad del Valle de Guatemala (UVG). Las entrevistas fueron grabadas a través de la plataforma Zoom, almacenadas en Google Drive. Para recolectar la información se utilizaron una guía de análisis 
documental y una entrevista semiestructurada basadas en un árbol de categorías, entre las cuales se contempla: 1. Participación de la población estudiantil con NEAE, 2. Elementos necesarios para la inclusión, 3. Diseño universal del aprendizaje, 4. Detección de NEAE previo a la ejecución de ajuste, 5. Contenidos, objetivos y evaluación inclusivos, 6. Construcción de significado y valoración de contexto, 7. La oralidad como punto de partida para acceder a la escritura, y 8 . Valoración de las hipótesis de lectoescritura de la población estudiantil. El punto de partida fue el análisis, las entrevistas se utilizaron para complementar y aclarar los hallazgos obtenidos mediante la aplicación de la primera técnica.

\subsection{Procesamiento de análisis}

3.4.1 Fase 1: Análisis documental del currículo y las guías de ajustes curriculares para la población estudiantil con NEAE

Los documentos se analizaron mediante una guía de análisis documental multinivel realizada en una hoja de cálculo, la cual consistía en un árbol de dimensiones y categorías, con indicadores que definían cada una. La guía de análisis posee tres grandes dimensiones que, a su vez, se dividen en categorías (ocho en total), cada una con sus respectivas subcategorías. En la Tabla 1 se plantean las dimensiones, categorías y subcategorías, y se refleja la relación que existe entre ellas. 
Tabla 1.

Árbol de categorías para el análisis de documentos curriculares para enseñanza de la LEI en República Dominicana

\begin{tabular}{|c|c|c|}
\hline Dimensión & Categorías & Subcategorías \\
\hline \multirow[t]{2}{*}{$\begin{array}{lr}\text { Paradigmas } & \text { pre } \\
\text { en las } & \text { prop } \\
\text { curriculares } & \text { pa } \\
\text { población } & \text { estı } \\
\text { con NEAE } & \end{array}$} & $\begin{array}{l}\text { - Participación de la población } \\
\text { estudiantil con NEAE }\end{array}$ & $\begin{array}{l}\text { - Exclusión } \\
\text { - Segregación } \\
\text { - Integración } \\
\text { - Inclusión }\end{array}$ \\
\hline & $\begin{array}{l}\text { - Elementos necesarios para la } \\
\text { inclusión }\end{array}$ & $\begin{array}{ll}\text { - } & \text { Mismo currículo } \\
\text { - } & \text { Flexibilidad del currículo } \\
\text { - } & \text { Ajuste curricular } \\
& \text { individualizado } \\
\text { - } & \text { Medidas de atención a la } \\
& \text { diversidad }\end{array}$ \\
\hline \multirow{3}{*}{$\begin{array}{l}\text { Concepción de } \\
\text { herramientas didácticas } \\
\text { en la enseñanza para la } \\
\text { población estudiantil } \\
\text { con NEAE }\end{array}$} & - Diseño universal del aprendizaje & $\begin{array}{l}\text { - Diseño universal del } \\
\text { aprendizaje }\end{array}$ \\
\hline & $\begin{array}{l}\text { - Detección de NEAE previo a la } \\
\text { ejecución de ajuste }\end{array}$ & $\begin{array}{l}\text { - Evaluación de NEAE previo } \\
\text { a la aplicación de ajustes } \\
\text { - Evaluación diagnóstica } \\
\text { - Evaluación de los } \\
\text { aprendizajes }\end{array}$ \\
\hline & $\begin{array}{l}\text { - Contenidos, objetivos y evaluación } \\
\text { inclusivos }\end{array}$ & $\begin{array}{l}\text { - } \quad \text { Contenidos y objetivos } \\
\text { - Evaluación de los } \\
\text { aprendizajes } \\
\text { - Estrategias de evaluación } \\
\text { - Instrumento flexible }\end{array}$ \\
\hline \multirow[t]{3}{*}{$\begin{array}{l}\text { Proceso } \\
\text { lectoescritura inicial de } \\
\text { la población estudiantil } \\
\text { con NEAE }\end{array}$} & $\begin{array}{l}\text { - Construcción de significado y } \\
\text { valoración de contexto }\end{array}$ & $\begin{array}{l}\text { - La construcción de } \\
\text { significado } \\
\text { - Valoración del contexto }\end{array}$ \\
\hline & $\begin{array}{l}\text { - La oralidad como punto de partida } \\
\text { para acceder a la escritura }\end{array}$ & $\begin{array}{l}\text { - Enseñanza de la lengua en } \\
\text { planos oral y escrito } \\
\text { - Fomento del uso de la } \\
\text { oralidad para llegar a la } \\
\text { escritura } \\
\text { - Conciencia fonológica }\end{array}$ \\
\hline & $\begin{array}{l}\text { - Valoración de las hipótesis de } \\
\text { lectoescritura de la población } \\
\text { estudiantil }\end{array}$ & - Etapas de adquisición \\
\hline
\end{tabular}

Fuente: Elaboración propia (2021). 


\subsubsection{Fase 2: Análisis de entrevistas semiestructuradas}

Las entrevistas fueron realizadas a través de la plataforma Zoom. El instrumento de levantamiento de la entrevista se diseñó a partir del marco de referencia producto del análisis documental basado en las tres grandes dimensiones que a su vez se dividieron en categorías y subcategorías. Constó de 17 preguntas abiertas y fue validado mediante juicio de 5 expertos con experiencia en lectoescritura inicial y educación inclusiva. Posterior a su realización, la información fue clasificada en una hoja de cálculo.

\subsubsection{Fase 3: Triangulación de los datos}

Una vez concluida la construcción del marco teórico, el análisis documental y la transcripción de las entrevistas, los datos se exportaron al programa de análisis cualitativo MAXQDA 2020 donde fueron codificados. Al finalizar este proceso se generaron matrices, mapas, listas de frecuencia y cuadrículas para la triangulación de los datos.

\section{Resultados}

Este apartado responde a la pregunta acerca de la alineación de los documentos curriculares y las guías de ajustes curriculares frente a la evidencia científica. En la Tabla 2 se muestra la presencia o ausencia de las categorías de análisis en cada uno de los textos revisados. 
Tabla 2.

Presencia de las categorías de análisis en los documentos curriculares dominicanos

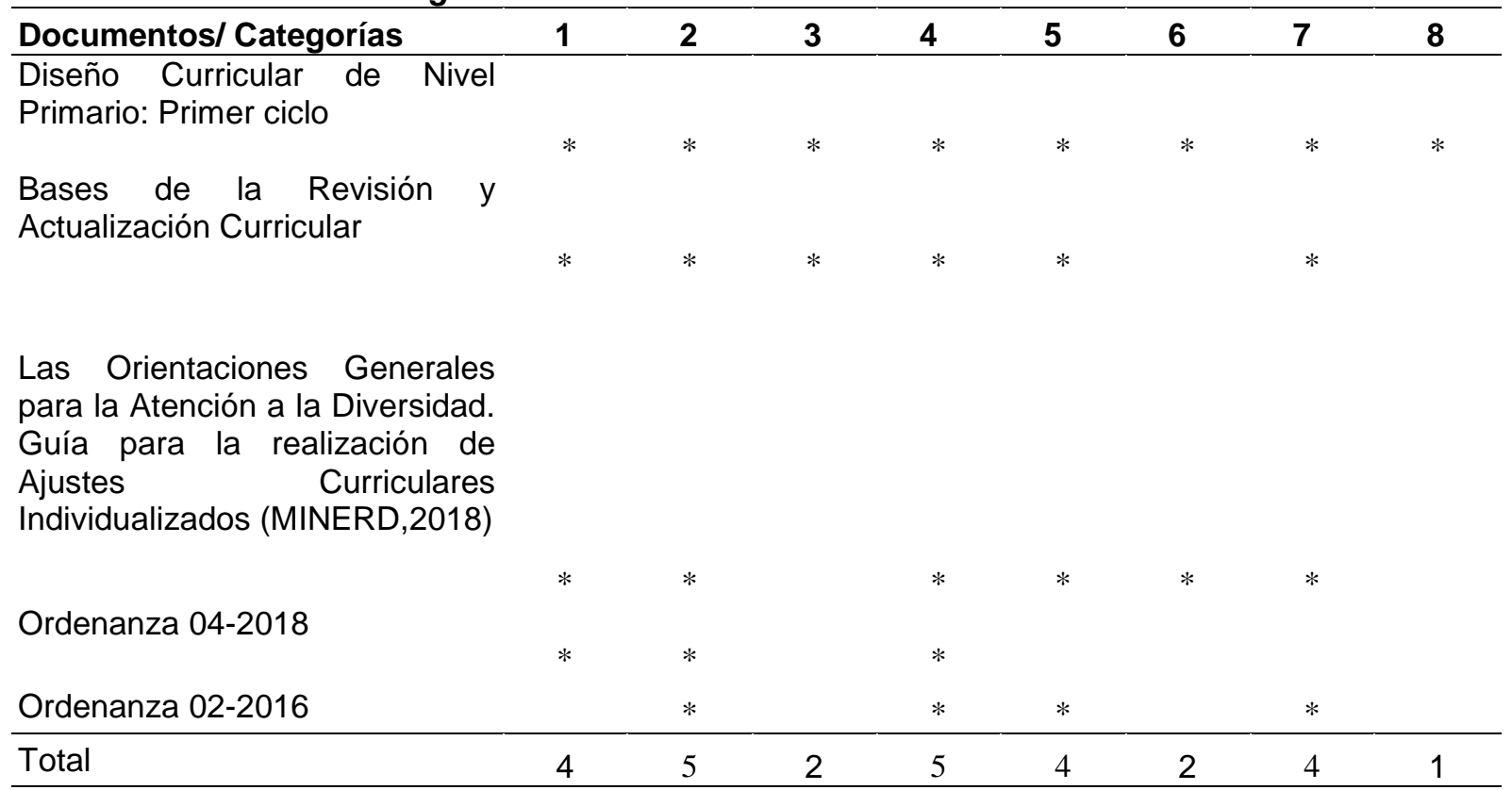

Nota: 1-Participación de la población estudiantil con NEAE 2-Currículo inclusivo 3-Diseño universal del aprendizaje 4-Detección de NEAE previo a la ejecución de ajuste 5-Contenidos, objetivos y evaluación inclusivos 6-Construcción de significado y valoración de contexto 7-La oralidad como punto de partida para acceder a la escritura 8-Valoración de las hipótesis de lectura y escritura de la población estudiantil

Fuente: Elaboración propia basada en el análisis (2021).

Se puede observar que en el currículo dominicano se identificaron todas las categorías, mientras que en las Ordenanzas se observó la mitad o menos, lo que denota baja alineación. En lo que respecta a las guías de ajustes, hay poca o nula presencia específica de LEI. Para continuar respondiendo a la pregunta, se desarrollarán los resultados obtenidos por dimensiones.

\subsection{Paradigmas presentes en las propuestas curriculares para la población estudiantil con NEAE}

La evidencia recopilada indica que las propuestas curriculares deben ser inclusivas, al alinear esto con las propuestas curriculares para la inclusión de la población estudiantil con NEAE se identificó que tanto la Ordenanza 04-2018 como MINERD (2018) se refieren a aulas inclusivas presentes dentro de las escuelas para apoyar los aprendizajes del estudiantado con NEAE, aunque frente a lo planteado, fomentan integración y no inclusión. Esto se constata en las entrevistas, las personas participantes afirman que el estudiantado con NEAE recibe clases 
dentro y fuera del aula en algunos momentos junto a sus compañeros y en otros en aulas especiales, práctica que responde a la integración. No se hallaron evidencias de exclusión en el análisis documental, tampoco en las entrevistas sobre las propuestas de cada uno de los documentos.

En lo que se refiere al paradigma de segregación, MINERD (2018) afirma que existen "Centros Específicos de Educación Especial" (p.33), lo que evidencia presencia de este enfoque. Sobre la integración, el mismo documento cita la Ordenanza 1-95 y su misión de "integrar a los alumnos con necesidades educativas al programa regular" (p.23). Además, confirma la existencia de Centros de Educación Especial cuando indica que estos pueden movilizarse hacia la educación regular cuando sea posible.

Frente a la evidencia, los mismos textos también contienen afirmaciones inclusivas, por su parte, MINERD (2018) plantea que "el desafío es crear una escuela y un entorno didáctico en los cuales se considere la diversidad como una oportunidad para el enriquecimiento de la clase y no como algo que entorpece el aprendizaje" (p.13). Esta idea es una de las que más se alinea a la evidencia hallada.

Tal como se ha observado hasta el momento, los documentos analizados incorporan diversos paradigmas. Un ejemplo que ilustra esto es el artículo 9 de la Ordenanza 04-2018, que indica diversos entornos de escolarización en los que se requiere participación del estudiantado con NEAE: escuelas especiales y regulares. Esto representa un reto para alcanzar la inclusión y la diversidad extendidas en todos los espacios educativos, así lo postulan García-Llamas (2008); Leiva (2013) y Molina (2015). En un tono más esperanzador, Luque (2009) refiere que los programas de integración deberían seguirse aplicando mientras se necesiten para convertirse en un mecanismo para alcanzar la inclusión, lo que quiere decir que la integración representa evolución frente a paradigmas de exclusión y segregación.

En lo que se refiere a que la población estudiantil con NEAE aprenda con el currículo nacional, tanto los cinco documentos analizados como la mayoría de personas participantes afirman que la población con NEAE está recibiendo formación con el mismo currículo que el resto del estudiantado. Esto se pudo observar cuando MINERD (2018) indica que "las escuelas específicas de Educación Especial implementan el mismo Diseño Curricular de los Centros Educativos del Nivel Inicial y Primario" (p. 34). Estas afirmaciones coinciden con los planteamientos de García-Llamas (2008) en los que refiere que para que un currículo sea inclusivo debe ser el mismo para todos, pero a la vez responden a un paradigma de segregación al tratarse de escuelas específicas. 
Morán y Álvarez (2013) afirman que, en los currículos flexibles, el estudiantado se vuelve guía experto con mayor libertad para actuar. Asimismo, se resalta que es indispensable que un currículo sea flexible para que se puedan tomar medidas de atención a la diversidad (García-Llamas, 2008; Valencia-Pérez y Hernández-González, 2017; Valladares et al., 2016). En alineación con esto, MINERD (2018) se refiere más de una vez a la necesidad de que el currículo sea flexible al momento de aplicar ajustes o atender a la diversidad. Igualmente, la mayor parte de las personas participantes afirmó que el currículo dominicano es flexible a adaptaciones curriculares. Otras dos personas participantes negaron esto utilizando expresiones como que "es muy rígido", y que el orden descendente desde la competencia al indicador inflexibiliza el currículo frente a la atención a la diversidad.

Otro aspecto abordado por la evidencia se refiere al empleo de los ACI. La Ordenanza 04-2018 (2018) plantea que "los Ajustes Curriculares Individualizados se realizan cuando el estudiante muestra un desempeño curricular con dos años o más de discrepancia entre el grado que le corresponde según su edad y sus competencias curriculares" (Artículo 2, párrafo 2). Este planteamiento responde a la integración, donde el estudiantado comparte el currículo, pero no logra inclusión plena, pues no se aplican medidas de atención a la diversidad. Acerca de estas, los documentos plantean que el estudiantado debe recibir un trato diversificado y particular acorde a sus necesidades específicas. MINERD (2018) destaca que:

Las medidas para la atención a la diversidad se elaboran sobre la base del diseño curricular dominicano concebido como el documento normativo que establece una estrategia para la formación de sujetos democráticos y transformadores de la realidad material, social y cultural. (p. 27)

Los cinco documentos analizados indican que las medidas de atención a la diversidad pueden ser de distintos caracteres y se desarrollan para evitar las barreras. Estos planteamientos se apegan a las afirmaciones de los autores referenciados en el marco teórico (Arnaiz, 2011; Parrilla et al. 2012; Stainback y Stainback, 1999).

\subsection{Concepción de herramientas didácticas para la enseñanza a la población estudiantil con NEAE}

La mayor parte de categorías referentes a las herramientas didácticas estuvieron presentes en los documentos analizados. En lo que se refiere al DUA, enfoque asumido en las premisas teóricas de esta investigación, en MINERD (2018) se pudieron observar algunos de 
sus principios y postulados, sin embargo, en los cuatro documentos restantes no se halló de manera explícita. La mayoría de la población entrevistada se refirió a este como la alternativa necesaria para lograr la inclusión en la enseñanza de la LEI; en consonancia con las ideas de Valencia-Pérez y Hernández-González (2017), quienes afirman que este modelo educativo se ocupa de la población estudiantil en sentido general, pues parte de la diversidad como una norma.

Un elemento indispensable para determinar la necesidad de un ajuste es la detección de una NEAE, todos los documentos analizados indican que la población infantil con NEAE requiere toda la atención de un equipo multidisciplinario que le apoye y supla sus necesidades dentro y fuera del aula. Además de referirse a evaluaciones diagnósticas, abordan la evaluación de los aprendizajes como un pilar de suma importancia, afirmación que coincide con Dabdub-Moreira y Pineda-Cordero (2015), quienes plantean que es sumamente importante que la persona docente regule estos procesos a fin de que el estudiantado reciba las atenciones educativas que le corresponden por derecho.

Otra herramienta didáctica presente en todo proceso educativo es la evaluación diagnóstica, el MINERD (2016a) le atribuye particular importancia cuando indica que "utilizan los resultados de la evaluación para reorientar su enseñanza y los aprendizajes de los niños y niñas" (p.102). Asimismo, MINERD (2016b) afirma que permite mejorar los procesos educativos. Además, MINERD (2018) habla de esta evaluación como el primer paso; mientras que la Ordenanza 02-2016 en su artículo 34, párrafo 1 plantea que las NEAE se van a determinar después de este tipo de evaluación y que debe ser realizada por un equipo multidisciplinario. Por su parte, la 04-2018 destaca que a partir de esta evaluación se indicarán los apoyos que necesite el estudiantado.

Continuando con las herramientas didácticas, se observaron los contenidos y objetivos en el área de Lengua Española, al respecto, MINERD (2016a) establece y describe una serie de criterios para seleccionar y organizar los contenidos (concepción, diferencias individuales, etapas de desarrollo, entre otros). En el resto de los textos no se reportan orientaciones acerca del ajuste de los contenidos para la LEI de la población estudiantil con o sin NEAE. Algo que se esperaría ver, como explican Espada y Moreno (2016), son los aspectos importantes para adaptar contenidos y sus características para que sean aptos para atender la diversidad (tomar en cuenta tamaño y color de letras, así como el volumen de voz en la comunicación) para la población estudiantil con NEAE. 
Otra herramienta indispensable en todo proceso educativo es la evaluación de los aprendizajes, la Ordenanza 02-2016 postula todas las indicaciones para evaluar a la población estudiantil con NEAE en sentido general y sin especificar por área curricular. Establece que la responsabilidad concierne al personal docente y del área de psicología, y la finalidad de la evaluación es la equidad como principio normativo. Aborda, además, los criterios de promoción y registro de ajustes en el sistema a nivel nacional. Por su parte, MINERD (2016b) afirma que la evaluación debe ser coherente con las intenciones curriculares. MINERD (2018) cuestiona la visión tradicional de la evaluación de los aprendizajes e indica que desde la inclusión esta se utiliza para identificar los recursos de apoyo que requiere el estudiantado. Estas afirmaciones coinciden con García-Llamas (2008), quien afirma que la evaluación debe ser cooperativa y alineada a los objetivos y contenidos.

Acerca de las estrategias de evaluación de los aprendizajes, todos los documentos analizados contienen planteamientos acerca de las diferentes estrategias para evaluar según la diversidad del estudiantado. La Ordenanza 02-2016 plantea que las evaluaciones para el estudiantado con NEAE deben contemplar procedimientos diversos. MINERD (2016a) sigue esa línea y plantea que es deber del personal docente utilizar diversidad de instrumentos (registro anecdótico, rúbricas, entre otros). También, MINERD (2016b) concibe la evaluación de los aprendizajes como una identificación de los logros. Además, MINERD (2018) afirma que los resultados nunca deben ser utilizados para clasificar o etiquetar al cuerpo estudiantil.

La población entrevistada mencionó instrumentos dispuestos por la Dirección General de Educación Especial, entre ellos un informe que les orienta qué fichas aplicar; sin embargo, la mayoría resaltó que estos no son específicamente de LEI. Los documentos curriculares recomiendan evitar pruebas estandarizadas, basarse en el rendimiento individual y utilizar las evaluaciones con fines formativos. La evaluación de los aprendizajes se suscribe a la literatura planteada; pero no se hallaron planteamientos específicos para la LEI (Gómez, 2018; Stainback y Stainback, 1999; Puigdellívol, 1998).

\subsection{El proceso de lectoescritura inicial de la población estudiantil con NEAE}

El enfoque que sustenta la enseñanza aprendizaje de la Lengua Española en República Dominicana es el funcional comunicativo, parte de la premisa de que los usuarios de la lengua se comunican mediante la construcción de significado y que las prácticas tienen siempre un fin. También plantea que las prácticas del lenguaje siempre deben tener un contexto. Respecto a eso, MINERD (2016a) propone el uso de situaciones de aprendizajes o circunstancias, 
puesto que estas se crean simulando la realidad para que el estudiantado pueda aplicar sus conocimientos. Acerca de la LEI, MINERD (2016a) plantea su enfoque con las siguientes palabras:

La lectura y la escritura se asumen desde el enfoque centrado en la construcción del significado y en la comunicación... La enseñanza de la lectura y la escritura es siempre guiada por unos propósitos definidos y destinatarios reales en un contexto específico, tal como ocurre en la vida. (p. 75)

Las personas entrevistadas coincidieron en que las guías de ajustes no plantean contenidos específicos de LEI, una de las personas participantes se refirió a aspectos puntuales, afirmando que se observa "el uso de pictogramas y el fomento de la competencia comunicativa”. MINERD (2018) tiene lineamientos generales y estos no responden a la LEI. En el ámbito de la lengua, Chaves (2001) confiere gran importancia a la asistencia a escuelas regulares a favor del aprendizaje de la lengua escrita.

Otro ámbito que indican las evidencias es la importancia de la enseñanza de la lengua en planos oral y escrito, en el MINERD (2016a) y la Ordenanza 02-2016 se observaron planteamientos al respecto, mientras que, aunque MINERD (2018) contiene recomendaciones generales referentes al uso de la lengua, no se refiere particularmente a la enseñanza de la LEI para la población con NEAE. La ordenanza previamente referida postula que se requiere que la población estudiantil haya completado el proceso de LEI antes de pasar a cuarto de primaria, por su parte, MINERD (2016a) desarrolla la construcción de significados.

Aunque la Participante A (comunicación personal, 12 marzo 2020) afirma que "lo primero es trabajar en estas áreas que son básicas para el desarrollo de las competencias orales y escritas, para el aprendizaje de la lectura y la escritura" y las guías se aproximan a la lengua brindando orientaciones acerca de la presentación de informaciones lingüísticas y no lingüísticas de manera efectiva, no se encontraron contenidos referidos específicamente a la LEI. Varias personas participantes afirmaron la necesidad de los ajustes y medidas para atender el aprendizaje de la lengua, pues indican que es una de las mayores necesidades del estudiantado con NEAE.

En el caso del fomento del uso de la oralidad para llegar a la escritura no se reportan hallazgos en el Diseño Curricular ni las Bases para la revisión y actualización curricular. Mientras que en MINERD (2018) solo se halló la recomendación de "realizar pruebas orales cuando el/la estudiante presente dificultades para escribir" (p.60). Respecto al vacío señalado, 
el currículo se enfoca más específicamente en la oralidad y la escritura al afirmar que en este nivel académico la población estudiantil avanza del lenguaje oral al escrito y se desarrolla mediante la interacción con sus cercanos; asimismo indica, que "la oralidad es de vital importancia en el proceso de construcción del conocimiento, ya que facilita la negociación de significado y de conceptos, el intercambio de informaciones, el análisis de problemas, la discusión y propuesta de soluciones posibles" (p.76). En ese mismo ámbito, los autores consultados confieren gran importancia a la conciencia fonológica como transición de la oralidad a la escritura (Caballeros et al., 2014; Quiroga, 2010).

Respecto a las etapas de adquisición de la LEI por las que atraviesa la población estudiantil para su adquisición, MINERD (2016a) afirma que:

Durante el proceso de construcción del sistema de escritura, los niños y las niñas atraviesan por diferentes etapas de apropiación, desde la consideración del dibujo como representación del significado, uso de letras o signos para representar sonidos hasta descubrir el principio alfabético de la escritura. (p.76)

Además, indica que la LEI se aprende en contacto con textos completos. Respecto a este indicador tampoco se reportan hallazgos específicos de LEl. Se le confiere gran importancia a estas hipótesis por ser el inicio de la fonetización (Ferreiro, 2019).

Las categorías previamente abordadas en conjunto con la Tabla 2 dejan constar cuán alineadas se encuentran las propuestas curriculares para la enseñanza de la LEI a la población estudiantil con NEAE. En las conclusiones se dejará ver de manera más general el contraste de la evidencia frente a los resultados hallados.

\section{Conclusiones}

\subsection{Paradigmas presentes en las propuestas curriculares para la población estudiantil con NEAE}

En esta dimensión se concluye que los documentos fomentan la participación del estudiantado con NEAE en la escuela desde varios paradigmas, entre los cuales no se encuentra la exclusión, pero sí la segregación (presente en las escuelas especiales cuya existencia se afirma en los documentos), la integración (en la participación de parte de la población estudiantil de manera parcial y el empleo de los ajustes en casos en los que se propondrían medidas de atención a la diversidad) y la inclusión en los momentos en los que se practica el DUA (García-Llamas, 2008; Leiva, 2013; Molina 2015; UNESCO, 2008). Esta 
diversidad de paradigmas es un reto a superar, sin embargo, el hecho de que haya presencia de inclusión es un rasgo positivo y sienta las bases de la eliminación futura de otros paradigmas.

Sobre el currículo y la inclusión es posible detectar que tanto el análisis documental como las entrevistas demostraron que se está utilizando el mismo currículo para toda la población estudiantil, incluso cuando están segregados, integrados o incluidos. Respecto a la flexibilidad del currículo, se identificaron diversas posiciones tanto en los textos como en las personas participantes, lo que representa un reto de reflexión y reinvención.

Un aspecto favorable y coherente con el DUA que se pudo verificar es que los textos analizados priorizan las medidas de atención a la diversidad y conciben el ajuste como una medida que procede después de haber agotado estrategias para atender la diversidad (Arnaiz, 2011; Parrilla et al. 2012; Stainback y Stainback, 1999).

\subsection{Concepción de herramientas didácticas para la enseñanza a la población estudiantil con NEAE}

En lo que se refiere al DUA, se pudo constatar que las guías de ajustes curriculares se rigen por la mayor parte de sus principios. Aunque sigue siendo un reto lograr el paradigma de la inclusión y reducir la segregación.

Respecto a la Detección de NEAE, previo a la ejecución de ajustes, se observó coherencia entre lo postulado por los autores y lo contenido en los textos analizados. Se podría afirmar lo mismo de la promoción de evaluaciones diagnósticas y psicopedagógicas.

En lo que se refiere a los contenidos de LEI y la inclusión frente a la literatura consultada, se juzga de positivo que el Diseño Curricular Dominicano proponga una serie de textos que coinciden en todos los grados del ciclo, esto permite medidas de atención a la diversidad más justas que propician la inclusión (Espada y Moreno, 2016). En la filosofía de la evaluación, los planteamientos son coherentes con el DUA, sin embargo, queda abierta la pregunta de si los indicadores de logro son idóneos para evaluar en ambientes en los que predomina la diversidad.

\subsection{El proceso de lectoescritura inicial de la población estudiantil con NEAE}

En MINERD (2018) hubo pocos hallazgos para esta dimensión. Esto se debe a que es un documento general para atender a la diversidad de toda la población estudiantil. Por su parte, en lo que se refiere a MINERD (2016a) y MINERD (2016b) se pudo observar 
alineamiento con el enfoque funcional comunicativo y textual. En estos documentos se visualizó el fomento de la oralidad para llegar a la escritura, la conciencia fonológica y las etapas de adquisición. Sin embargo, el vacío de estas temáticas en las guías representa un reto para la elaboración de un documento con medidas de atención a la diversidad enfocadas en la población infantil con NEAE. Cabe destacar que la interacción con las personas entrevistadas y la revisión bibliográfica demuestran que este documento suple una serie de necesidades y que su formulación puede representar una gran motivación para especializarse por área curricular o por ciclo educativo. Antes de finalizar, conviene resaltar que las categorías relacionadas a la LEI no estuvieron presentes en las guías de ajustes curriculares.

\subsection{Ideas finales}

Para concluir conviene regresar al inicio y preguntar ¿Cuán alineados se encuentran el currículo y sus guías de ajustes con lo que indica la evidencia respecto a la LEI de la población estudiantil con necesidades específicas de apoyo educativo? Se podría afirmar que los indicadores se cumplen de manera parcial, lo que representa un reto para lograr la inclusión y que esta permita que la población estudiantil aprenda a leer y escribir acorde a su nivel, ritmo y capacidad. También se concluye que se necesita una propuesta de ajustes curriculares que responda a las necesidades específicas de la población infantil.

En lo que concierne a las limitaciones del estudio, por ser de alcance meramente bibliográfico, no se observó la aplicación en las escuelas debido a las limitaciones producto de la Pandemia del COVID-19. Esta decisión se fundamentó en que las clases a distancia no eran una representación de las presenciales y para evitar el contacto presencial, que podría comprometer la salud de posibles personas participantes y las investigadoras. Se propone una futura investigación que valore el nivel de inclusión de la población estudiantil con NEAE en las aulas dominicanas con el fin de constatar la aplicación de lo planteado teóricamente por los documentos.

\section{Agradecimientos}

A la Red para la Lectoescritura Inicial de Centroamérica y el Caribe (RedLEI), por el acompañamiento continuo y sistemático, la entrega, disposición y apoyo durante el desarrollo de esta investigación. También, a Carmen García, directora del Centro de Excelencia para la Difusión e Investigación de la Lectura y Escritura (CEDILE) de la Pontificia Universidad Católica Madre y Maestra (PUCMM), por el seguimiento y la asesoría a lo largo de este proceso. 


\section{Referencias}

Arnaiz, Pilar. (2012). Escuelas eficaces e inclusivas: cómo favorecer su desarrollo. Educatio Siglo XXI, 30(1), 25-44. https://revistas.um.es/educatio/article/view/149121

Báez, Mónica., y D’Ottavio, María Eugenia. (2020). La diversidad en el aula: el desafío de interpretar la singularidad de los procesos de alfabetización inicial. Ciencia y Educación, 3(3), 31-40. https://doi.org/10.22206/cyed.2019.v3i3

Braslavsky, Berta. (2000). Las nuevas perspectivas de la alfabetización temprana. Lectura y vida, 21(4), 32-43. http://www.lecturayvida.fahce.unlp.edu.ar/numeros/a21n4/21 04 Braslavsky.pdf

Brennan, Wilfred. (1985). El currículo para niños con necesidades especiales. España: Siglo $\mathrm{XXI}$.

Caballeros, Marta Zoila., Sazo, Eva., y Gálvez, José Andrés. (2014). El aprendizaje de la lectura y escritura en los primeros años de escolaridad: experiencias exitosas de Guatemala. Interamerican Journal of Psychology, 48(2), 212-222. https://doi.org/10.30849/rip/ijp.v48i2.261

Castillo, Gineida., y Mola, José. (2016). Tercer estudio regional comparativo y explicativo 2013 (Informe nacional República Dominicana). Santo Domingo, República Dominicana: Ministerio de Educación de República Dominicana.

Chall, Jeanne Sternlicht. (1983). Stages of Reading Development. New York: McGraw-Hill.

Chaves, Ana Lupita. (2001). La apropiación de la lengua escrita: un proceso constructivo, interactivo y producción cultural. Revista Actualidades Investigativas en Educación, 1(1). DOI: 10.15517/aie.v1i1.8455

Corredor-Ponce, Zulema. (2016). Las adecuaciones curriculares como elemento clave para asegurar una educación inclusiva. Educación en contexto, 2(3), 56-78. https://dialnet.unirioja.es/descarga/articulo/6296699.pdf

Dabdub-Moreira, Mayela., y Pineda-Cordero, Alejandra. (2015). La atención de las necesidades educativas especiales y la labor docente en la escuela primaria. Revista Costarricense de Psicología, 58. https://dialnet.unirioja.es/servlet/articulo?codigo=5278249

Espada, Rosa., y Moreno, Ricardo. (2016). Adaptaciones curriculares: bases y estructuración desde la experiencia Universitaria. CienciAmérica, 5(1), 126128. http://cienciamerica.uti.edu.ec/openjournal/index.php/uti/article/view/60

Ferreiro, Emilia. (2019). Research perspectives in beginning literacy. Methodological and epistemic dichotomies/Prospectivas de la investigación en la alfabetización inicial. Dicotomías metodológicas y epistémicas. Infancia y Aprendizaje, 42(1), 1-36. https://doi.org/10.1080/02103702.2018.1550174 
García-Llamas, José Luis. (2008). Aulas inclusivas. Bordón: Revista de Pedagogía, 60(4), 89105. https://recyt.fecyt.es/index.php/BORDON/article/view/28871

Gómez, Jorge. (2018). Correlation. En Inclusión educativa y adaptaciones curriculares en el proceso de enseñanza aprendizaje (pp. 91-118). Ecuador: Editorial UTMACH.

Guirado, Vania y Guerra, Sonia. (2013). Recursos didácticos y sugerencias metodológicas para la enseñanza-aprendizaje de los escolares con necesidades educativas especiales. Segunda parte. La Habana: Pueblo y Educación.

Jaramillo, Claudia. (2018). Diseño de estrategias lúdico-pedagógicas para enseñanza lectoescritura a estudiantes con necesidades especiales de aprendizaje. Educación y Territorio, 8(14), 29-48. https://dialnet.unirioja.es/servlet/articulo?codigo=7993902

Leiva, Juan José. (2013). De la integración a la inclusión: Evolución y cambio en la mentalidad del alumnado universitario de educación especial en un contexto universitario español. Revista Electrónica Actualidades Investigativas en Educación, 13(3). https://www.scielo.sa.cr/scielo.php?script=sci arttext\&pid=S1409-47032013000300025

León, Juan. (2018). Programa Internacional para la Evaluación de los Estudiantes PISA 2015: Informe Nacional. Santo Domingo, República Dominicana: Ministerio de Educación de República Dominicana.

López, Isabel., y Valenzuela, Gloria. (2015). Niños y adolescentes con necesidades educativas especiales. Revista Médica Clínica Condes, 26(1), 42-51. Doi https://doi.org/10.1016/i.rmclc.2015.02.004

Lores-Leyva, Idevis., y Calzadilla-González, Onaida. (2013). Los trastornos de la lectura y la escritura en escolares de la Educación Primaria. EduSol, 13(42), 13-23. https://dialnet.unirioja.es/servlet/articulo?codigo=5678513

Luque, Diego Jesús. (2009). Las necesidades educativas especiales como necesidades básicas. Una reflexión sobre la inclusión educativa. Revista Latinoamericana de Estudios Educativos, 39(3-4), 201-223. https://www.redalyc.org/pdf/270/27015078009.pdf

Luque, Diego Jesús., y Luque, María Jesús. (2015). Alumnado con necesidades específicas de apoyo educativo: aspectos psicopedagógicos en un marco inclusivo. Perspectiva Educacional, Formación de Profesores, 54(2), 59-73. https://www.redalyc.org/articulo.oa?id=333339872005

Ministerio de Educación de la República Dominicana (MINERD). (2016a). Diseño Curricular Nivel Primario: Primer Ciclo. Santo Domingo, República Dominicana: Ministerio de Educación de la República Dominicana.

Ministerio de Educación de la República Dominicana (MINERD). (2016b). Bases de la Revisión y Actualización Curricular. Santo Domingo, República Dominicana: Ministerio de Educación de la República Dominicana. 
Ministerio de Educación de la República Dominicana (MINERD). (2018). Orientaciones Generales para la Atención a la Diversidad. Guía para la realización de Ajustes Curriculares Individualizados (ACl). Santo Domingo, República Dominicana: Ministerio de Educación de la República Dominicana.

Molina, Yasna. (2015). Necesidades educativas especiales, elementos para una propuesta de inclusión educativa a través de la investigación acción participativa. El caso de la Escuela México. Estudios Pedagógicos, 41(especial), 147-167. http://dx.doi.org/10.4067/S0718$\underline{07052015000300010}$

Morán, Lourdes., y Álvarez, Guadalupe. (2013). Currículum abierto y flexible: análisis del caso de una materia universitaria con integración de TIC. Revista electrónica de tecnología educativa, (45). https://dialnet.unirioja.es/servlet/articulo?codigo=5746376

Montealegre, Rosalía., y Forero, Luz Adriana. (2006). Desarrollo de la lectoescritura: adquisición y dominio. Acta Colombiana de Psicología, 9(1), 25-40. http://www.scielo.org.co/scielo.php?pid=S012391552006000100003\&script=sci abstract\&tIng=es

Ocampo, Aldo y Calero, Andrés. (2018). Competencia Lectora y Comprensión Lectora desde una perspectiva de Educación inclusiva. Entrevista a Andrés Calero. Revista Electrónica de Investigación Educativa, 20(3), 1-12. https://redie.uabc.mx/redie/article/view/2186

Ordenanza 02-2016. (2016). Establece el sistema de evaluación de los aprendizajes en la Educación inicial y primaria en correspondencia con el currículo revisado y actualizado. 19 de diciembre del 2016. Santo Domingo, República Dominicana: Ministerio de Educación de República Dominicana.

Ordenanza 04-2018. (2018). Norma los servicios y estrategias para el cuerpo estudiantil con necesidades específicas de apoyo educativo acorde al currículo. 26 de julio del 2018. Santo Domingo, República Dominicana: Ministerio de Educación de República Dominicana.

Parrilla, Ángeles., Martínez, Esther., y Zabalza, María Ainoa. (2012). Diálogos infantiles en torno a la diversidad y la mejora escolar. Revista Educación, 359, 120-142. DOI:10.4438/1988-592X-RE-2012-359-199

Paz-Delgado, Carla., y Barahona, Elma. (2020). Creencias y prácticas docentes sobre la inclusión educativa en el contexto de aprendizaje de la lectoescritura inicial. Revista Caribeña de Investigación Educativa (RECIE), 4(2), 112-126. https://doi.org/10.32541/recie.2020.v4i2.pp112-126

Puigdellívol, Ignasi. (1998). La Educación Especial en la escuela integrada: una perspectiva desde la diversidad. Barcelona, España: Editorial Graó.

Quiroga, Patricia. (2010). La evolución de la atención a la diversidad del alumnado de educación primaria a lo largo de la historia. Rev. Dig. Prof. Ens, 8, 1-15. https://feandalucia.ccoo.es/docu/p5sd7241.pdf 
Robson, Colin. (2005). Students with disabilities, learning difficulties and disadvantages: Statistics and indicators. Organisation for Economic Cooperation and Development (OCDE).

Ruiz Andrés, Roberto. (2020). Dificultades y contradicciones en la respuesta educativa para los alumnos con necesidad específica de apoyo educativo (ACNEAE). Aula (26), 149168. https://doi.org/10.14201/aula202026149168

Stainback, Susan., y Stainback, William. (1999). Aulas inclusivas. Un nuevo modo de enfocar y vivir el currículo. México: Narcea Ediciones.

Teberosky, Ana. (2003). Alfabetización inicial: aportes y limitaciones. Cuadernos De Pedagogía, 330, 42-46. https://dialnet.unirioja.es/servlet/articulo?codigo=760304

UNESCO. (2008). La educación inclusiva: El camino hacia el futuro. En Conferencia Internacional de Educación. Ginebra.

UNESCO. (2017). Guía para asegurar la inclusión y la equidad en la educación. París: UNESCO.

UNESCO. (2019). Educación inclusiva para personas con discapacidades: ¿Estamos logrando avances? En el Foro Internacional sobre Inclusión y Equidad en la Educación «Todas y todos los estudiantes cuentan», Cali, Colombia.

Valencia-Pérez, Cristina., y Hernández-González, Osvaldo. (2017). El Diseño Universal para el Aprendizaje, una alternativa para la inclusión educativa en Chile. Atenas, 4(40), 105120. https://www.redalyc.org/articulo.oa?id=478055150008

Valladares, María Angélica., Betancourt, Ingrid., y Norambuena, Mariela. (2016). Correlation. En XI y XII jornadas de cooperación educativa con iberoamérica sobre Educación especial e Inclusión educativa. París: UNESCO.

Warnock, Mary. (1978). Special educational needs. Informe del Commitee of Inquiry into Education of Handicapped Children and Young People. H. M. S. O. Her Majesty's Stationery Office. 
Revista indizada en

scit/o redalyc latindex

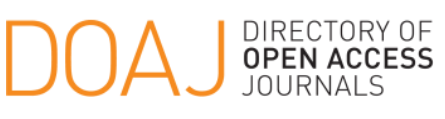

Distribuida en las bases de datos:
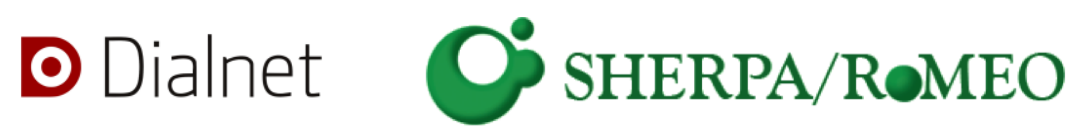

REDIB

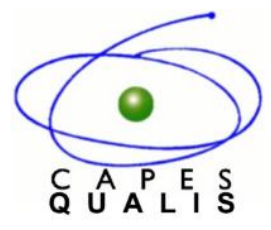

MIAR 